\title{
Empleo de ventanas de escape en trampas para la captura de jaiba peluda (Cancer setosus) en Iquique, Chile
}

\author{
Mario Aguilar $^{1}$ \& Pedro Pizarro ${ }^{1}$ \\ ${ }^{1}$ Departamento de Ciencias del Mar, Universidad Arturo Prat \\ Casilla 121, Iquique, Chile
}

\begin{abstract}
RESUMEN. Se analizó la inclusión de ventanas de escape en trampas para la extracción de jaiba peluda (Cancer setosus Molina, 1782) en la pesquería de jaibas en la zona norte de Chile. Se diseñaron y construyeron trampas de modelo tronco-cónico con entrada superior, adicionando ventanas de escape en la base de formas circular y rectangular, y un tercer modelo sin ventanas de escape, a modo de control, para comprobar la utilidad del escape. Estas trampas se instalaron en una línea de pesca con 12 unidades a una profundidad entre 5 y $8 \mathrm{~m}$. Las operaciones de pesca se efectuaron mensualmente, cerca del puerto de Iquique. La CPUE, medida en número de individuos por trampa, fue mayor en el modelo sin ventanas de escape; sin embargo, si se considera sólo los ejemplares iguales o mayores a 120 $\mathrm{mm}$, la suma de la captura de las trampas con ventanas de escape representó el 59,8\% de la captura total. Los datos se analizaron mediante un test de ANOVA de una vía para la captura total y por sexo. Los resultados indicaron que no hay diferencias significativas en la talla media del ancho cefalotoráxico entre los diferentes modelos de trampas, como tampoco al separar este análisis respecto de los sexos ni cuando se considera a los individuos mayores a la talla legal de captura. Dado los resultados obtenidos, es recomendable que en futuras investigaciones se considere el tiempo de reposo, tamaño y número de ventanas por trampa.
\end{abstract}

Palabras clave: trampas, ventanas de escape, Cancer setosus, jaiba peluda, Chile.

\section{The use of escape windows in crab (Cancer setosus) traps in Iquique, Chile}

\begin{abstract}
The use of escape windows in traps set for the crab (Cancer setosus Molina, 1782) was analyzed in the crab fishery in the north of Chile. Troncoconic trap models with top entrances were designed and manufactured in order to test the use of escape windows. One model included circular escape windows at the bottom and the other rectangular escape windows; the third model (control) had no escape windows. These traps (12 units) were installed on a fishing line at depths between 5 and $8 \mathrm{~m}$. The fishing operations were done monthly, near the Iquique port. The CPUE, measured as the number of individuals per trap, was greater in the model without escape windows. However, when only considering specimens of $120 \mathrm{~mm}$ or larger, the combined catch from the traps with escape windows made up $59.8 \%$ of the total catch. The data were analyzed using a one-way ANOVA test for the total catch and by sex. The results indicate no significant differences in the average size of the carapace width between the different trap models, not even when doing the analysis separately by sex or when considering the individuals exceeding the legal catch size. Given the results obtained, it is recommendable that future research considers the soaking time, size, and number of windows per trap.
\end{abstract}

Key words: traps, escape windows, Cancer setosus, crab, Chile.

Autor corresponsal: Mario Aguilar (maguilar@unap.cl)

\section{INTRODUCCIÓN}

La selectividad se define como la propiedad de los artes de pesca de capturar recursos de determinado rango de tallas y especie de una población; no obstante, en el caso de algunos artes pasivos, como las trampas, no existe seguridad respecto a cuál sería su comportamiento en la pesca de crustáceos. Por dicha razón, es importante encontrar formas de retener las tallas mayores o legales sin tener que posteriormente, efectuar un manejo de las capturas a bordo de las embarcaciones para devolver al mar 
los ejemplares de talla pequeña.

Para mejorar la selectividad de las trampas se han efectuado investigaciones enfocadas preferentemente a modificar o buscar nuevos diseños que impidan la entrada o faciliten el escape de las tallas menores o sublegales. Los estudios se han realizado cambiando el tamaño de las mallas que cubren las paredes de las trampas (Guillary \& Prejean, 1997; Guillary, 1998; Nishiuchi, 2003); evaluando la inclusión de ventanas de escape (Arana \& Díaz, 1987; Arana \& Ziller, 1994; de la Rosa-Pacheco \& Ramírez-Rodríguez, 1996; Nishiuchi, 2003); probando el tamaño y la forma de los escapes (Everson et al., 1992; Arana \& Ziller, 1994; Zhou \& Shirley, 1997; Atar et al., 2002); analizando la posición de los escapes respecto de la base (Nishiuchi, 2003); modificando los diseños comerciales de las trampas existentes (Zhou \& Kruse, 2000); estudiando el tamaño y ubicación de la boca de entrada (Yamane \& Fujiishi, 1992); incluyendo elementos adicionales como anillos plásticos de diferentes alturas en la entrada de la trampa (Herbert et al., 2001). En todas las investigaciones se manifiesta la necesidad de disminuir la cantidad capturada de tallas sublegales como su manejo a bordo, reduciendo los tiempos de operación, así como los daños y mortalidades de los individuos pequeños, sin interés comercial para los pescadores.

En crustáceos, las reglamentaciones por lo general definen la talla legal de captura respecto de sus dimensiones morfométricas, sexo o condición reproductiva. En Chile, se prohíbe la comercialización de jaiba peluda o pachona (Cancer setosus) cuyo ancho cefalotoráxico (AC) sea menor a 120 $\mathrm{mm}$ y de hembras que portan huevos en el abdomen (ovígeras).

La jaiba peluda se distribuye desde el golfo de Guayaquil (Ecuador) hasta la península de Taitao (Chile). En la zona norte de Chile, su extracción se realiza mediante buceo semi-autónomo (hooka) empleando embarcaciones de $7 \mathrm{~m}$ de eslora, equipadas usualmente con motores fuera de borda de $25 \mathrm{HP}$, en faenas de pesca que se efectúan durante el día, a profundidades entre 2 y $15 \mathrm{~m}$. Aguilar et al. (1999) estudiaron la introducción de trampas en la pesquería de jaibas, evaluando tres diseños: tronco-cónico, tronco-piramidal y paralelogramo rectangular, los dos primeros con entradas superiores y sin dispositivos de no retorno (von Brandt, 1984), y el tercero con entradas laterales en forma de túnel rectangular. Dicha investigación permitió determinar que el modelo tronco-cónico presenta la mejor opción de operación, tanto por sus resultados de CPUE $\left(\mathrm{N}^{\circ}\right.$ de ejemplares trampa ${ }^{-1}$ ) como por la posibilidad de apilamiento, esto permite usar un elevado número de trampas sin requerir de gran espacio en la cubierta de la embarcación.

Complementariamente y ya elegido el mejor modelo, era necesario establecer su habilidad selectiva. Por esta razón, se experimentó en el empleo de trampas con ventanas de escape y sin ellas, con el objeto de determinar la retención de las mismas respecto al ancho del cefalotórax de los ejemplares.

\section{MATERIALES Y MÉTODOS}

Para este estudio se diseñaron y construyeron trampas tronco-cónicas (Aguilar et al., 1999). Las dimensiones de las trampas correspondieron a base de $80 \mathrm{~cm}$ de diámetro, altura de $35 \mathrm{~cm}$ y boca de $20 \mathrm{~cm}$ de diámetro. Se incluyeron en la base ventanas de escape de dos tipos: circular de $100 \mathrm{~mm}$ de diámetro y rectangular de $100 \times 50 \mathrm{~mm}$, conservando un tercer diseño sin dispositivos de escape, que actuó como trampa control y comparación respecto a los dispositivos de selectividad aplicados en el presente estudio (Fig. 1). Las dimensiones de los escapes se seleccionaron conforme a las relaciones morfométricas obtenidas por Aguilar et al. (1999).

Se efectuaron 12 cruceros de pesca experimental entre Mejillones del Norte (19\%49'S, $\left.70^{\circ} 09^{\prime} \mathrm{W}\right)$ e Iquique $\left(20^{\circ} 12^{\prime} \mathrm{S}, 70^{\circ} 10^{\prime} \mathrm{W}\right)$ (Fig. 2), con lances de 5 a $8 \mathrm{~m}$ de profundidad, entre octubre de 2002 y septiembre de 2003. Las trampas se distribuyeron en líneas (tenas) de 12 trampas, con el siguiente orden: trampa de control, ventana circular y ventana rectangular, repetido cuatro veces, con una separación entre nasas de $18 \mathrm{~m}$ (Fig. 3). A bordo de la embarcación los ejemplares capturados fueron separados por tipo de trampa. Posteriormente, en el laboratorio, a cada ejemplar se le registró el sexo, ancho y longitud cefalotóraxica con un vernier a 1 $\mathrm{mm}$, diferenciando los sexos de acuerdo a la morfología del abdomen.

Se calculó la captura por unidad de esfuerzo (CPUE), en número de individuos capturados por tipo de trampa. Se evaluó las diferencias en las capturas por modelo de trampa respecto al ancho cefalotóraxico tanto a nivel global como por sexo, y para tallas iguales o mayores de $120 \mathrm{~mm}$. Los datos se analizaron y compararon mediante ANOVA de una vía a un nivel de significancia $\alpha=0,05$ mediante el programa computacional STATGRAPHIC $+\mathrm{v} 2,1$. 


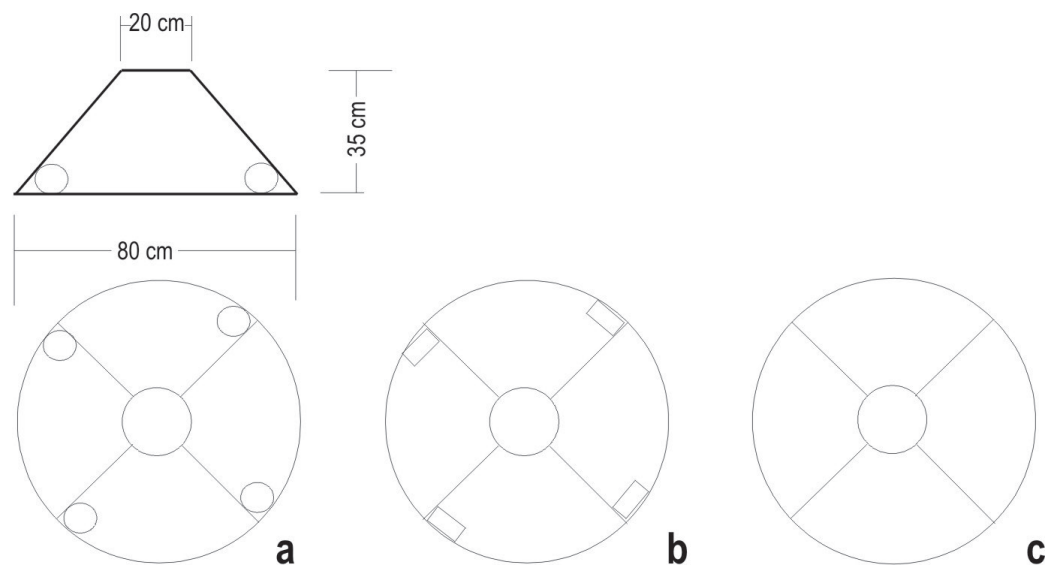

Figura 1. Trampas tronco-cónicas con ventanas de escape: a) circular, b) rectangular y c) trampas de control, puestas a prueba para la pesca de jaiba peluda (Cancer setosus).

Figure 1. Truncated cone traps with escape vents: a) circular, b) rectangular, and c) control trap, for fishing of hairy crab (Cancer setosus).

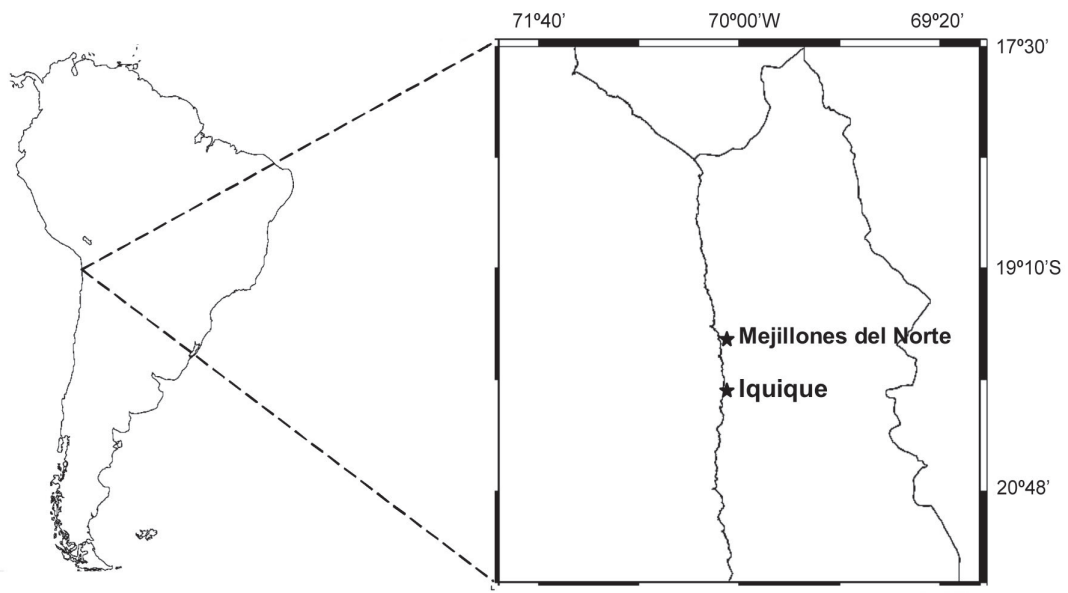

Figura 2. Zona de pesca experimental frente a Iquique, Chile.

Figure 2. Area of experimental fishing in Iquique, Chile.

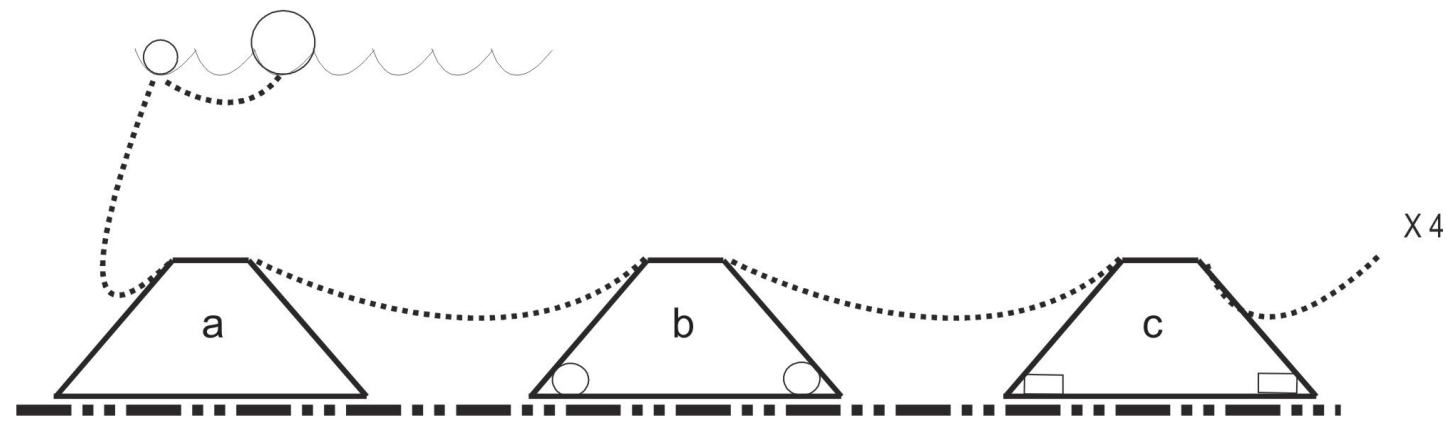

Figura 3. Disposición de las trampas en los lances de pesca experimental. a) control, b) escape circular y c) escape rectangular.

Figure 3. Traps disposition in the experimental fishing. a) control, b) circular vent, and c) rectangular vent. 


\section{RESULTADOS}

Se calaron 144 trampas y se capturó un total de 558 jaibas cuyo ancho cefalotoráxico varió entre 47 y $144 \mathrm{~mm}$, de las cuales el $68,3 \%$ correspondió a hembras. En hembras se determinó un AC medio de $102,0 \pm 12,9 \mathrm{~mm}$; en machos de 106,3 $\pm 17,3$ $\mathrm{mm}$ y en hembras ovígeras de $99,1 \pm 8,5 \mathrm{~mm}$. Con tallas superiores a $120 \mathrm{~mm}$ de AC se capturaron 82 individuos que representaron el $14,7 \%$ de la captura total; no se observó predominio de un sexo respecto al otro, excepto que las tallas de los machos fueron ligeramente mayores (Tabla 1).

Respecto a las capturas por tipo de trampa (en número de individuos), se determinó que un 48,9\% correspondió a la trampa control, y un $23,1 \%$ y $27,9 \%$ a los aparejos con escape circular y con ventana rectangular, respectivamente (Tabla 2). Se observó que las capturas por sexo y tipo de trampa fueron mayores en las trampas sin dispositivos de escape. No obstante, al considerar las tallas medias, las trampas con ventanas de escape circular retuvieron individuos mayores, tanto hembras (102 $\pm 14,1$ $\mathrm{mm})$ como machos $(108 \pm 17,7 \mathrm{~mm})$. Igualmente, al comparar el rango de tallas se detectó que las tallas mínimas de las trampas con ventanas de escape fueron superiores a la establecida en las trampas sin escape (Tabla 2).

Los resultados de la captura por unidad de esfuerzo (CPUE) medido en número de individuos por trampa muestran que tanto para el total de ejemplares como por sexo, los valores son mayores para la trampa sin ventanas de escape, mientras que la que utiliza escapes en forma rectangular se sitúa en

Tabla 1. Número, porcentaje y rango de ancho cefalotoráxico (AC) de jaiba peluda por sexo para el total capturado y $\mathrm{AC} \geq 120 \mathrm{~mm}$.

Table 1. Number, percentage and range of carapace width (AC) of hairy crab by sex for the total catch and AC $\geq 120 \mathrm{~mm}$.

\begin{tabular}{clcccc}
\hline & Sexo & Número & \% & $\begin{array}{c}\text { Mínimo } \\
(\mathbf{m m})\end{array}$ & $\begin{array}{c}\text { Máximo } \\
(\mathbf{m m})\end{array}$ \\
\hline \multirow{2}{*}{ AC } & Hembras & 373 & 66,9 & 66 & 143 \\
(captura total) & Machos & 177 & 31,7 & 47 & 144 \\
& Hembras ovígeras & 8 & 1,4 & 88 & 115 \\
\hline \multirow{2}{*}{$\mathbf{A C}$} & Hembras & 40 & 48,8 & 120 & 143 \\
$\mathbf{1 1 2 0}$ & Machos & 42 & 51,2 & 120 & 144 \\
& Hembras ovígeras & 0 & 0,0 & & \\
\hline
\end{tabular}

Tabla 2. Número, media, desviación estándar (DE) y rango de ancho cefalotoráxico (AC) de jaiba peluda por sexo y por tipo de trampa para el total capturado y $\mathrm{AC} \geq 120 \mathrm{~mm}$.

Table 2. Number, mean, standard deviation (DE) and range of carapace width (AC) of hairy crab by sex and by trap type for total catch and $\mathrm{AC} \geq 120 \mathrm{~mm}$.

\begin{tabular}{clrrrrcc}
\hline & Trampa & $\mathbf{N}^{\mathbf{0}}$ & $\mathbf{\%}$ & $\begin{array}{c}\text { Media } \\
(\mathbf{m m})\end{array}$ & $\begin{array}{c}\mathbf{D E} \\
(\mathbf{m m})\end{array}$ & $\begin{array}{c}\text { Mínimo } \\
(\mathbf{m m})\end{array}$ & $\begin{array}{c}\text { Máximo } \\
(\mathbf{m m})\end{array}$ \\
\hline $\begin{array}{c}\text { AC } \\
\text { (captura total) }\end{array}$ & Escape circular & 129 & 23,12 & 104,98 & 15,50 & 70 & 143 \\
& Escape rectangular & 156 & 27,96 & 102,65 & 14,09 & 71 & 144 \\
& Control & 273 & 48,92 & 103,02 & 14,32 & 47 & 141 \\
\hline \multirow{2}{*}{$\mathbf{A C}$} & Escape circular & 30 & 36,59 & 126,77 & 6,70 & 120 & 143 \\
$\geq \mathbf{1 2 0}$ & Escape rectangular & 19 & 23,17 & 128,16 & 6,38 & 120 & 144 \\
& Control & 33 & 40,24 & 127,03 & 5,79 & 120 & 141 \\
\hline \multirow{2}{*}{ Hembras } & Escape circular & 86 & 22,57 & 102,15 & 14,12 & 73 & 143 \\
& Escape rectangular & 104 & 27,30 & 101,50 & 12,10 & 73 & 132 \\
& Control & 191 & 50,13 & 101,00 & 12,67 & 66 & 137 \\
\hline \multirow{3}{*}{ Machos } & Escape circular & 43 & 24,29 & 108,43 & 17,66 & 70 & 141 \\
& Escape rectangular & 52 & 29,38 & 105,76 & 17,04 & 71 & 144 \\
& Control & 82 & 46,33 & 105,60 & 17,41 & 47 & 141 \\
\hline
\end{tabular}


segundo lugar (Tabla 3).

Si se considera sólo los aparejos modificados y sólo los ejemplares de $\mathrm{AC} \geq 120 \mathrm{~mm}$, la trampa con ventana de escape circular, presenta los mejores porcentajes de captura de individuos legales (Fig. 4), tanto para el total de ejemplares, como separados por sexo (Tabla 3). Si se considera la CPUE entre las trampas que emplean ventanas, los mayores valores corresponden a la trampa con escape circular, tanto respecto al total como por sexo (Tabla 3). Sumada la CPUE de las trampas con ventanas de escape, estas representan el 59,8\% de las capturas de individuos legales, mientras que el restante 40,2\% corresponde a las trampas control (Tabla 3 ).

El análisis de ANOVA en Cancer setosus para el total de la captura muestra que no hay diferencias significativas entre trampas $(F=1,06 ; p=0,348)$. Al separar este análisis respecto del sexo; se obtiene un rechazo de la hipótesis de existencia de capturas diferenciadas para hembras $(F=0,66 ; p=0,519)$ y machos $(F=0,42 ; p=0,660)$. Respecto de las tallas legales $(\mathrm{AC} \geq 120 \mathrm{~mm})$ no se detectan diferencias estadísticamente significativas entre trampas para el total de las capturas $(\mathrm{F}=0,31 ; \mathrm{p}=0,738)$ como tampoco cuando se analizan para hembras $(\mathrm{F}=0,51 ; \mathrm{p}$ $=0,603)$ y machos $(\mathrm{F}=0,13 ; \mathrm{p}=0,874)($ Tabla 4$)$.

\section{DISCUSIÓN}

Los estudios sobre selectividad están orientados a permitir el escape de las tallas sublegales y en el caso de los crustáceos, a disminuir el manejo tanto de tallas menores como también de hembras ovígeras. La posibilidad de incorporar ventanas de escape en las trampas para jaibas permitiría disminuir la manipulación de las tallas sublegales a bordo, tal como lo expresan Arana \& Díaz (1987), Guillary \& Prejaen (1997) y Atar et al. (2002). Además, impediría el traslado de éstas hacia otras zonas, dado que normalmente la clasificación se efectúa durante la navegación hacia el puerto de desembarque. Este método ha sido usado como una técnica para

Tabla 3. CPUE para el total capturado y con ancho cefalotoráxico $(\mathrm{AC}) \geq 120 \mathrm{~mm}$ por tipo de trampa.

Table 3. CPUE total catch and carapace width (AC),$\geq 120 \mathrm{~mm}$ by trap type.

\begin{tabular}{|c|c|c|c|c|c|c|c|c|}
\hline & \multirow[t]{2}{*}{ Trampa } & \multirow{2}{*}{$\begin{array}{l}N^{\circ} \text { de } \\
\text { trampas }\end{array}$} & \multicolumn{3}{|c|}{$\mathrm{N}^{0}$ individuos capturados } & \multicolumn{3}{|c|}{ 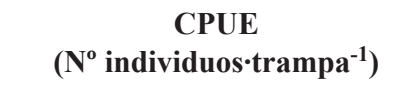 } \\
\hline & & & Total & Hembras & Machos & Total & Hembras & Machos \\
\hline \multirow{3}{*}{$\begin{array}{c}\text { AC } \\
\text { (captura } \\
\text { total) }\end{array}$} & Escape circular & 48 & 129 & 86 & 43 & 2,69 & 1,79 & 0,90 \\
\hline & Escape rectangular & 48 & 156 & 104 & 52 & 3,25 & 2,17 & 1,08 \\
\hline & Control & 48 & 273 & 191 & 82 & 5,69 & 3,98 & 1,71 \\
\hline \multirow{3}{*}{$\begin{array}{c}\mathrm{AC} \\
\geq 120\end{array}$} & Escape circular & 48 & 30 & 15 & 15 & 0,63 & 0,31 & 0,31 \\
\hline & Escape rectangular & 48 & 19 & 7 & 12 & 0,40 & 0,15 & 0,25 \\
\hline & Control & 48 & 33 & 18 & 15 & 0,69 & 0,38 & 0,31 \\
\hline
\end{tabular}

Tabla 4. Análisis de varianza de acuerdo al ancho cefalotoráxico (AC) de Cancer setosus, por sexo y trampas. g.l.: grados de libertad, F: estadístico, p: probabilidad.

Table 4. Analysis of variance of carapace width (AC) of Cancer setosus for sex and traps. gl: degrees of freedom, F: statistical, p: probability.

\begin{tabular}{clccccc}
\hline & Variable & $\begin{array}{c}\text { Suma de } \\
\text { cuadrados }\end{array}$ & gl & $\begin{array}{c}\text { Media } \\
\text { cuadrado }\end{array}$ & F & p \\
\hline \multirow{2}{*}{$\begin{array}{c}\text { AC } \\
\text { (captura total) }\end{array}$} & Total captura & 447,507 & 2 & 223,754 & 1,06 & 0,3476 \\
& Hembras & 217,393 & 2 & 108,696 & 0,66 & 0,5189 \\
& Machos & 251,419 & 2 & 125,710 & 0,42 & 0,6598 \\
\hline \multirow{2}{*}{$\mathbf{A C}$} & Total captura & 24,015 & 2 & 12,008 & 0,31 & 0,7377 \\
$\geq \mathbf{1 2 0}$ & Hembras & 30,661 & 2 & 15,330 & 0,51 & 0,6033 \\
& Machos & 12,536 & 2 & 6,268 & 0,13 & 0,8742 \\
\hline
\end{tabular}




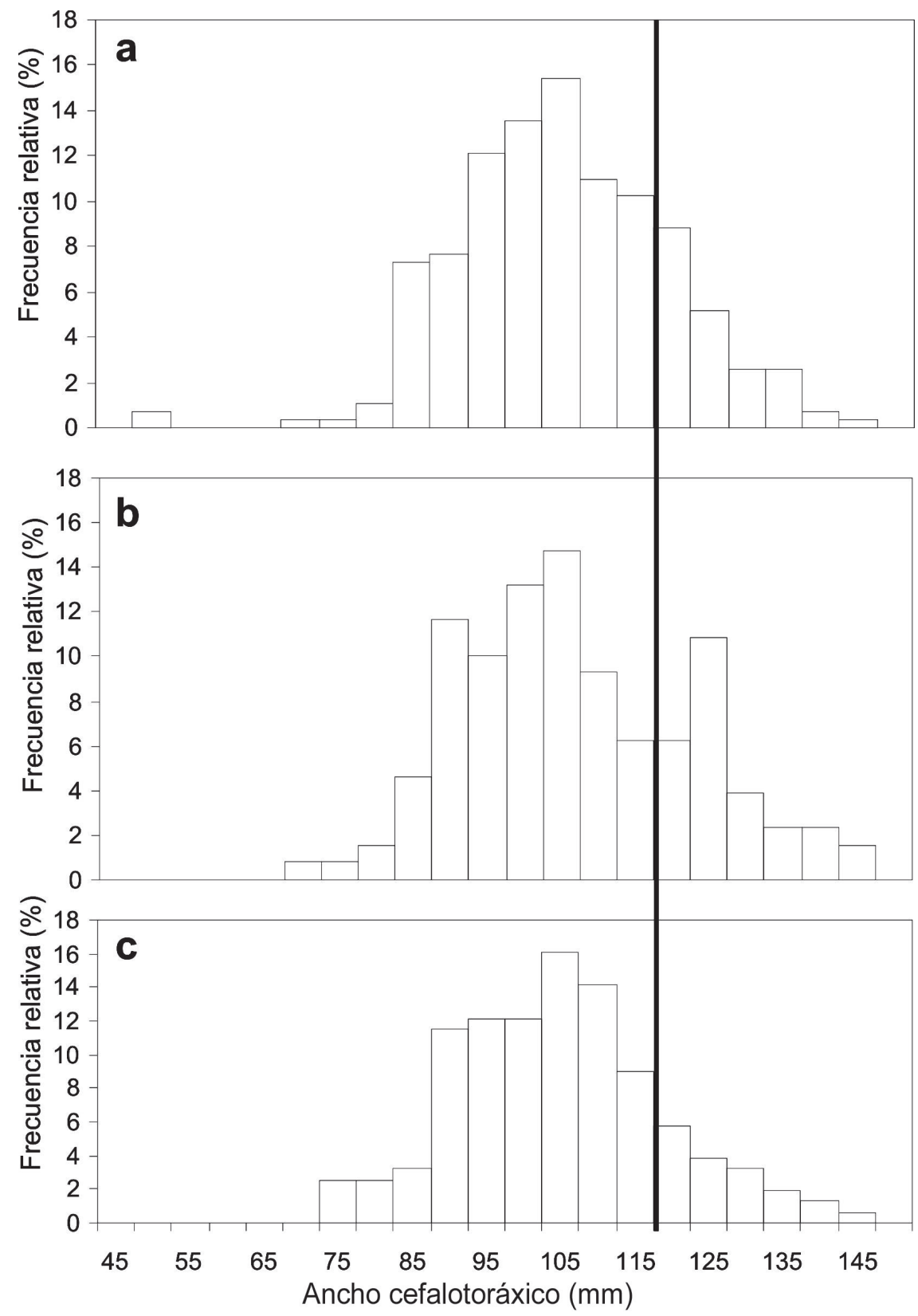

Figura 4. Frecuencia relativa de ancho cefalotoráxico (AC) para el total de la captura y por tipo de trampa: a) control, b) escape circular, y c) escape rectangular. Se indica $\mathrm{AC}=120 \mathrm{~mm}$ de cefalotórax.

Figure 4. Relative frequency of caparace width (AC) for the total catch by trap type: a) control trap, b) circular vents, and c) rectangular vents. Indicated $\mathrm{AC}=120 \mathrm{~mm}$. 
reducir la captura de tallas sublegales (Arana \& Díaz, 1987; Everson et al., 1992). De la Rosa-Pacheco y Ramírez-Rodríguez (1996) indican que el uso de ventanas de escape, independiente del tipo y diseño, conlleva a la disminución de la captura de tallas sublegales y tiende a mejorar la retención de las tallas legales. Similar sugerencia indican Herbert et al. (2001) quienes enfatizan la necesidad de disminuir la mortalidad por manejo de las tallas sublegales y la mantención del stock reproductivo mediante la incorporación de elementos de escape en las trampas. Esta investigación muestra que el uso de las ventanas de escape disminuye la captura de individuos de tallas sublegales y de hembras ovigeras, e incrementa la captura de jaibas de talla legal, respecto de la trampa que no tiene escapes, indicando así que los dispositivos utilizados serían un buen método para lograr significativos mejoramientos en la selectividad de las trampas, al igual como lo manifiesta Nishiuchi (2003).

En cuanto al tamaño de las ventanas, las de tipo circular $\left(0,008 \mathrm{~m}^{2}\right)$ brindan un área de escape de alrededor de $60 \%$ mayor respecto a las de tipo rectangular $\left(0,005 \mathrm{~m}^{2}\right)$; este diseño de escapes circulares sería más apropiado, ya que la selección se efectuaría tanto respecto a la altura del individuo como del ancho del cefalotórax, mientras que las de tipo rectangular limitarían su habilidad solo a la altura. Estos resultados concuerdan con Everson et al. (1992) quienes además indican que se posibilita el escape de especies taxonómicamente semejantes. Los resultados entre los dos tipos de escape muestran que las trampas de ventanas circulares reportan mejores capturas de tallas legales. Zhou \& Shirley (1997), indican que el ancho no sería una buena medida para el diseño de las aberturas de escape, concluyendo que las diferencias morfológicas proveen de un buen indicador para el diseño de los escapes y que debiera considerarse tanto el alto como el largo. Por otra parte, Arana \& Ziller (1994) señalan que también es necesario considerar el sexo y los apéndices corporales para determinar las dimensiones más apropiadas para las ventanas de escape. Yanane \& Fujiishi (1992) y Atar et al. (2002) sugieren que la búsqueda de los tamaños óptimos, tanto de las ventanas como de la entrada de la trampa, contribuiría a diferenciar las tallas de captura.

Es necesario considerar, tanto en futuras investigaciones como en pesca comercial, la habilidad de escape de los individuos, el tiempo de reposo de las trampas y el número de las ventanas de escape, tal como sugieren Zhou \& Kruse (2000) y Nishiuchi
(2003). Puesto que pareciera ser que, incrementando la permanencia de la trampa en el agua, aumentaría la probabilidad de escape de las tallas sublegales, la que se incrementaría manteniendo un mayor número de ventanas. Además, se debe considerar a futuro la búsqueda de dimensiones óptimas para las ventanas de escape considerando las relaciones morfométricas entre largo y ancho de cefalotórax.

El análisis de varianza (ANOVA) muestra que no existen diferencias en los AC en la captura de la jaiba peluda, sea evaluado tanto entre sexos como respecto al total, corroborado por la estructura de las tallas de los individuos capturados por los tres modelos de trampas que fueron comparados. Ahora bien, si se considera como patrón de comparación la CPUE en número de individuos de tallas legal capturados ( $\mathrm{AC} \geq 120 \mathrm{~mm}$ ), se percibe que existen diferencias, ya que se capturaron, sumados para ambas trampas, más ejemplares en los modelos con ventanas $(59,8 \%)$ que en la trampa control $(40,2 \%)$. Este último índice es reportado por Zhou \& Kruse (2000), quienes no encuentran diferencias entre las trampa estudiadas, como tampoco en la estructura de tallas en las capturas.

\section{AGRADECIMIENTOS}

El financiamiento para la realización de la investigación fue de la Dirección de Investigación de la Universidad Arturo Prat. Los autores agradecen a la tripulación de la L/M Antares señores Agustín Arcos y Claudio Vega por su colaboración en las pescas experimentales. Asimismo, los autores expresan su agradecimiento a los correctores anónimos por sus valiosas sugerencias.

\section{REFERENCIAS}

Aguilar, M., J.P. Díaz \& P. Pizarro. 1996. Las trampas de pesca, una alternativa de explotación de jaibas en la pesquería artesanal de la provincia de Iquique. Rev. Invest. Cien. Tecnol., Ser. Cien. Mar, 5: 70-75.

Arana, P. \& J. Díaz. 1987. Utilización de trampas con dispositivos de escape en la pesquería de langosta de Juan Fernández (Jasus frontalis). En: P. Arana (ed.). Manejo y desarrollo pesquero. Escuela de Ciencias del Mar, Universidad Católica de Valparaíso, Valparaíso, pp. 83-99.

Arana, P. \& S. Ziller. 1994. Modelación de la selectividad de trampas para la captura de langosta ( $\mathrm{Ja}$ - 
sus frontalis) en el archipiélago de Juan Fernández (Chile). Inv. Pesq. (Chile), 38: 1-21.

Atar, H., M. Ölmez, S. Becan \& S. Seçer. 2002. Comparison of three different traps for catching blue crab (Callinectes sapidus) in Beymelek Lagoon. Turk. J. Vet. Anim. Sci., 26: 1145-1150.

De la Rosa-Pacheco, R. \& M. Ramírez-Rodríguez. 1996. Ventanas de escape en trampas para la captura de la langosta roja Panulirus interreptus en Baja California Sur, México. Cienc. Mar., 22(2): 235-243.

Everson, A.R., R.A. Skillman \& J.J. Polovina. 1992. Evaluation of rectangular and circular escape vents in the northwestern Hawaiian Island lobster fishery. North. Am. J. Fish. Manage, 12: 161-171.

Guillary, V. \& P. Prejean. 1997. Blue crab, Callinectes sapidus, traps selectivity studies. Mar. Fish. Rev., 59(1): 29-31.

Guillary, V. 1998. Blue crab, Callinectes sapidus, retention rates in different trap meshes. Mar. Fish. Rev., 60 (1): 35-37.

Recibido: 20 julio 2005: Aceptado: 6 octubre 2006.
Herbert, M., G. Miron, M. Moriyasu, R. Vienneau \& P. DeGrace. 2001. Efficiency and ghost fishing of snow crab (Chionoecetes opilio) traps in the Gulf of St. Lawrence. Fish. Res., 52: 143-153.

Nishiuchi, S. 2003. A study on size selectivity of hair crabs pots. Sci. Rep. Hokkaido Fish. Exp. Stn., 64: 1-103.

Von Brandt, A. 1984. Fish catching methods of the world. Fishing News Books, Farham, Surrey, England, $418 \mathrm{pp}$.

Yamane, T. \& A. Fujiishi. 1992. Catch performance of small prawn pots in terms of selected and escape. Fish. Res., 15: 291-299.

Zhou, S. \& G. Kruse. 2000. Capture efficiency and size selectivity of two types of pots for red king crab in the Bering Sea. Alaska Fish. Res. Bull., 6(2): 94-103.

Zhou, S. \& T.C. Shirley. 1997. Performance of two red king crab pot designs. Can J. Fish. Aquat. Sci., 54: 1858-1864. 